\title{
EFFECT OF PACLOBUTRAZOL AND POTASSIUM NITRATE ON LEAVES NUTRIONAL STATUS OF EWAIS AND SIDIK MANGO TREES:
}

\section{2- Total sugars and amino acids}

Khattab, M.M.; G.M.M. Haseeb; A.E.A. Shaban and M.A. Arafa.

Pomology department, faculty of Agriculture, Cairo University, Egypt.

\begin{abstract}
This study was conducted throughout two seasons on grafted Ewais and Sidik mango trees grown at Elwadi El-Faregh Cairo/Alexandria desert road, Giza governorate Egypt. Trees were treated at the first week of October 2001 and 2002with potassium nitrate (KNO3) at 1,2 and $4 \%$ as a foliar spray, while Paclobutrazol (PBZ) was added at the first week of October 2001 at 2.5, 5 and $7.5 \mathrm{~g}$ per tree as a soil drench in 20 liter of water. Results indicated that both PBZ and KNO3 increased total soluble sugars in leaves compared to the control. A direct relationship was found between the used concentration and total sugars content. The highest total sugars content was recorded in Dec. then decreased gradually during Jan. and Feb. after that another increase occurred in Mar. PBZ and KNO3 significantly improved amino acids content in leaves of both cultivars. Increasing the used concentration of both chemicals significantly increased this content. Total amino acids increased from Nov. to Dec. then it decreased significantly during the period from Jan. to Mar. This was confirmed in both cultivars during the two seasons.

keywords: Mango (Mangifera indica L.)- Paclobutrazol- Potassium nitrate- Total sugars - Total amino acids.
\end{abstract}

\section{INTRODUCTION}

Mango (Mangifera indica L.) is considered one of the most important fruit crops in the world. There are many mango cultivars grown in Egypt such as Ewais and Sidik. Alternate bearing, low productivity of young mango trees are the most important problems facing mango production.

One of the most resources used in fruit crops and especially in mango is the application of growth regulators and flowering promoters such as growth retardants which used for its effect on controlling vegetative growth (Anez,2004). The growth retardant paclobutrazol which inhibits gibberellin biosynthesis has been successfully used to induce off- season flowering or to ensure uniform on season flowering of Nam Dok Mai mangoes (Tongumpai et al., 1989). Application of paclobutrazol enhanced mango flowering, increased the yield and improved fruit quality of Nam Dok Mai mango cV ( Tongumpai et al. ,1997). Potassium nitrate (KNO3) was used to enhance flowering and tree yield in mango( Sharma et al. 1990, Oosthuyse 1993 and 1996). Growth retardant affect the nutritional status of shoots and leaves by improving their minerals content which gave a positive effect on inducing flowering.

Consequently, this work was conducted to investigate the effects of paclobutrazol and potassium nitrate on total sugars and amino acids in leaves of Ewais and Sidik mango trees. 


\section{MATERIALS AND METHODS}

Ewais and Sidik mango trees were the plant material of this study which grown in a private orchard located at Elwadi El-Faregh Cairo/Alexandria desert road, Giza governorate, Egypt. The trees were about 5 years old on the start of the experiment. They were grafted on seedling rootstocks, planted in a sandy soil at $5 \times 5 \mathrm{~m}$ apart, under drip irrigation system. Chosen trees were uniform in growth vigor and received the same horticultural practices.

The experiment was conducted on the same selected trees during two consecutive seasons2001-2002 and 2002-2003. At the first season, trees were in the on-year and were off-year in second season. The completely randomized block design was used where each treatment was replicated three times, each comprising one tree.

Trees were treated at the first week of October 2001and 2002 (prefloral bud induction as determined by Zidan et al., 1975) with potassium nitrate (KNO3) at 1,2 and $4 \%$ as a foliar spray and Paclobutrazol (PBZ, Cultar) at 2.5, 5 and $7.5 \mathrm{~g}$ per tree as a soil drench in 20 liter of water. Paclobutrazole was added to the trees once at the first week of October 2001 as a soil drench, its effect was studied during both first and second seasons of investigation.

Nine leaves were taken from the upper, middle and lower parts of one year old shoots at monthly intervals from November to March for determination of total soluble sugars according to A.O.A.C. (1975) and total soluble amino acids according to Rosein (1957).

Data were tabulated and statistically analyzed according to Snedecor and Cochran (1980). Duncan multiple range tests were used to differentiate means at $5 \%$ level (Duncan, 1955).

\section{RESULTS AND DISCUSSION}

\subsection{Effect of PBZ and $\mathrm{KNO} 3$ on total soluble sugars content in leaves of Ewais and Sidik mangos:}

Data in tables $(1,2,3$ and 4) show the effect of PBZ and KNO3 on total sugars content in leaves of Ewais and Sidik cultivars. It is cleared that PBZ significantly increased total sugars in the leaves of Ewais cv compared to the control. A direct relationship was found between PBZ concentration and total sugars content as the highest values were recorded with $7.5 \mathrm{~g}$ per tree followed by 5 then $2.5 \mathrm{~g}$ per tree. The highest content of soluble sugars was recorded in Dec. This was confirmed in both seasons in PBZ treated trees and control. The same effect was noticed in Sidik cultivar except in the second season for the lowest level $2.5 \%$.

Concerning KNO3 treatments, results indicated that total sugar content in leaves of Ewais cultivar significantly decreased with application of $\mathrm{KNO} 3$ at the lower levels (1 and $2 \%$ ) in the first season, meanwhile, it increased significantly with $\mathrm{KNO} 3$ at $4 \%$. At the second season spraying $\mathrm{KNO} 3$ at 2 or $4 \%$ was significantly more effective in increasing total sugars content than the 
lowest level (1\%) and control. The highest total sugars content was recorded in Dec. with $\mathrm{KNO} 3$ at all levels and control trees in both seasons.

As for Sidik cultivar, KNO3 at 2 and $4 \%$ significantly increased total sugars compared to the control. Meanwhile, the lowest level of $\mathrm{KNO} 3$ was significantly less effective than control. A direct relationship was found between $\mathrm{KNO} 3$ concentrations and sugars content. It is noted that the highest total sugars content was detected in Dec. in both seasons either in KNO3 treated or control trees.

Concerning the changes in total sugars regardless of treatments, results showed a pronounced increase in Dec. then decreased gradually during Jan. and Feb., after that another increase was occurred in Mar. The accumulation of sugars during Dec. may be important for flower bud differentiation in this period and the second gradual increase in March may due to its relation to bud burst and flowering. This may help in overcoming the alternate bearing phenomenon in these cultivars by the application of PBZ at 5 or $7.5 \mathrm{~g}$ per tree one time in October every two years or KNO3 at 2 or $4 \%$ as spray every year in October. These findings confirmed the results obtained on the same cultivars as $\mathrm{PBZ}$ and $\mathrm{KNO} 3$ treatments increased the percentage of flowered shoots Khattab et al.(2006). Shaban (2004) studied seasonal changes of leaves sugars content in Zebda mango cv, he found that total sugars tend to increase gradually and significantly from October to December, thereafter it showed a gradual decrease from January to March.

Table (1): Effect of PBZ and KNO3 on total soluble sugars content ( $\mathrm{mg} / \mathrm{g}$ f.w.) in leaves of Ewais mango cv. (first season).

\begin{tabular}{|c|c|c|c|c|c|c|c|c|}
\hline \multirow{2}{*}{$\begin{array}{c}\text { Chemical } \\
\text { Substances }\end{array}$} & \multirow{2}{*}{ Conc. } & \multicolumn{5}{|c|}{ Date of sampling } & \multirow{2}{*}{ Mean } & \multirow{2}{*}{ Mean } \\
\hline & & Nov. & Dec. & Jan. & Feb. & Mar. & & \\
\hline \multirow{3}{*}{ PBZ (g/tree) } & 2.5 & $\begin{array}{c}9.05 \\
p\end{array}$ & $\begin{array}{c}9.92 \\
g\end{array}$ & $\begin{array}{c}9.83 \\
h\end{array}$ & $\begin{array}{c}9.71 \\
\mathrm{i}\end{array}$ & $\begin{array}{l}9.48 \\
\mathrm{Im}\end{array}$ & $\begin{array}{c}9.59 \\
D\end{array}$ & \multirow{3}{*}{$\begin{array}{c}10.01 \\
\mathbf{A}\end{array}$} \\
\hline & 5 & $\begin{array}{c}9.23 \\
\text { op }\end{array}$ & $\begin{array}{c}10.35 \\
b\end{array}$ & $\begin{array}{c}10.29 \\
\text { bc }\end{array}$ & $\begin{array}{c}10.26 \\
\text { cd }\end{array}$ & $\begin{array}{c}10.05 \\
\text { ef }\end{array}$ & $\begin{array}{c}10.03 \\
B\end{array}$ & \\
\hline & 7.5 & $\begin{array}{c}9.46 \\
\text { I-n }\end{array}$ & $\begin{array}{c}11.12 \\
a\end{array}$ & $\begin{array}{c}11.05 \\
a\end{array}$ & $\begin{array}{c}10.35 \\
b\end{array}$ & $\begin{array}{c}10.11 \\
\mathrm{e}\end{array}$ & $\begin{array}{c}10.42 \\
A\end{array}$ & \\
\hline \multirow{3}{*}{ KNO3 (\%) } & 1 & $\begin{array}{c}9.00 \\
r\end{array}$ & $\begin{array}{c}9.56 \\
\mathrm{jk}\end{array}$ & $\begin{array}{c}9.41 \\
\mathrm{mn}\end{array}$ & $\begin{array}{c}9.39 \\
n\end{array}$ & $\begin{array}{c}9.29 \\
0 \\
\end{array}$ & $\begin{array}{c}9.33 \\
\mathrm{G}\end{array}$ & \multirow{3}{*}{$\begin{array}{c}9.54 \\
\text { B }\end{array}$} \\
\hline & 2 & $\begin{array}{c}9.11 \\
9\end{array}$ & $\begin{array}{c}9.82 \\
h\end{array}$ & $\begin{array}{c}9.62 \\
j\end{array}$ & $\begin{array}{c}9.51 \\
\mathrm{kl}\end{array}$ & $\begin{array}{c}9.46 \\
I-n\end{array}$ & $\begin{array}{c}9.50 \\
F\end{array}$ & \\
\hline & 4 & $\begin{array}{c}9.18 \\
\mathrm{pq}\end{array}$ & $\begin{array}{c}10.21 \\
d\end{array}$ & $\begin{array}{c}10.00 \\
f\end{array}$ & $\begin{array}{c}9.83 \\
\mathrm{~h}\end{array}$ & $\begin{array}{c}9.73 \\
\mathrm{i}\end{array}$ & $\begin{array}{c}9.79 \\
\text { C } \\
\end{array}$ & \\
\hline \multicolumn{2}{|c|}{ Control } & $\begin{array}{c}9.12 \\
9\end{array}$ & $\begin{array}{c}9.86 \\
\text { gh }\end{array}$ & $\begin{array}{c}9.72 \\
\mathrm{i}\end{array}$ & $\begin{array}{c}9.61 \\
j\end{array}$ & $\begin{array}{c}9.45 \\
1-n\end{array}$ & $\begin{array}{c}9.55 \\
E\end{array}$ & $\begin{array}{c}9.55 \\
\text { B } \\
\end{array}$ \\
\hline \multicolumn{2}{|l|}{ Mean } & $\begin{array}{c}9.16 \\
E\end{array}$ & $\begin{array}{c}10.12 \\
\boldsymbol{A}\end{array}$ & $\begin{array}{c}9.99 \\
B\end{array}$ & $\begin{array}{c}9.81 \\
C\end{array}$ & $\begin{array}{c}9.65 \\
D\end{array}$ & & \\
\hline
\end{tabular}

Values followed by the same letter(s) are not significantly different at $5 \%$ level. 
Khattab M.M. et al.

Table (2): Effect of PBZ and $\mathrm{KNO} 3$ on total soluble sugars content ( $\mathrm{mg} / \mathrm{g}$ F.W.) in leaves of Ewais mango cv. (second season).

\begin{tabular}{|c|c|c|c|c|c|c|c|c|}
\hline \multirow[b]{2}{*}{ Treatments } & \multirow{2}{*}{ Conc. } & \multicolumn{5}{|c|}{ Date of sampling } & \multirow{2}{*}{ Mean } & \multirow{2}{*}{ Mean } \\
\hline & & Nov. & Dec. & Jan. & Feb. & Mar. & & \\
\hline \multirow{3}{*}{ PBZ (g/tree) } & 2.5 & $\begin{array}{c}10.12 \\
\mathrm{~s}\end{array}$ & $\begin{array}{c}10.92 \\
i\end{array}$ & $\begin{array}{c}10.81 \\
\mathrm{kl}\end{array}$ & $\begin{array}{c}10.13 \\
\mathrm{~s}\end{array}$ & $\begin{array}{c}9.86 \\
u\end{array}$ & $\begin{array}{c}10.37 \\
G\end{array}$ & \multirow{3}{*}{$\begin{array}{c}10.87 \\
\mathbf{A}\end{array}$} \\
\hline & 5 & $\begin{array}{c}10.36 \\
r\end{array}$ & $\begin{array}{c}11.06 \\
\text { gh }\end{array}$ & $\begin{array}{c}10.92 \\
i\end{array}$ & $\begin{array}{c}10.83 \\
k\end{array}$ & $\begin{array}{c}10.72 \\
\mathrm{~m}\end{array}$ & $\begin{array}{c}10.78 \\
\text { C }\end{array}$ & \\
\hline & 7.5 & $\begin{array}{c}11.25 \\
f\end{array}$ & $\begin{array}{c}11.85 \\
b\end{array}$ & $\begin{array}{c}11.72 \\
d\end{array}$ & $\begin{array}{c}11.46 \\
\mathrm{e}\end{array}$ & $\begin{array}{c}11.08 \\
\text { gh }\end{array}$ & $\begin{array}{c}11.47 \\
\text { A }\end{array}$ & \\
\hline \multirow{3}{*}{ KNO3 (\%) } & 1 & $\begin{array}{c}10.00 \\
t\end{array}$ & $\begin{array}{c}10.86 \\
\mathrm{jk}\end{array}$ & $\begin{array}{c}10.62 \\
n\end{array}$ & $\begin{array}{c}10.53 \\
p\end{array}$ & $\begin{array}{c}10.41 \\
\mathrm{qr}\end{array}$ & $\begin{array}{c}10.48 \\
F\end{array}$ & \multirow{3}{*}{$\begin{array}{c}10.84 \\
\text { B }\end{array}$} \\
\hline & 2 & $\begin{array}{c}10.39 \\
\mathrm{ar}\end{array}$ & $\begin{array}{c}11.03 \\
h\end{array}$ & $\begin{array}{c}10.91 \\
\text { ij }\end{array}$ & $\begin{array}{c}10.77 \\
\mathrm{Im}\end{array}$ & $\begin{array}{c}10.61 \\
\text { no }\end{array}$ & $\begin{array}{c}10.74 \\
D\end{array}$ & \\
\hline & 4 & $\begin{array}{c}11.09 \\
\mathrm{~g}\end{array}$ & $\begin{array}{c}11.91 \\
a\end{array}$ & $\begin{array}{c}11.79 \\
\mathrm{C}\end{array}$ & $\begin{array}{c}10.93 \\
\mathrm{i}\end{array}$ & $\begin{array}{c}10.82 \\
\mathrm{kl}\end{array}$ & $\begin{array}{c}11.31 \\
B\end{array}$ & \\
\hline \multicolumn{2}{|c|}{ Control } & $\begin{array}{c}10.15 \\
\mathrm{~s}\end{array}$ & $\begin{array}{c}10.89 \\
\text { ij }\end{array}$ & $\begin{array}{c}10.81 \\
\mathrm{kl}\end{array}$ & $\begin{array}{c}10.56 \\
\text { op }\end{array}$ & $\begin{array}{c}10.42 \\
9\end{array}$ & $\begin{array}{c}10.56 \\
E\end{array}$ & $\begin{array}{c}10.56 \\
\text { C }\end{array}$ \\
\hline \multicolumn{2}{|l|}{ Mean } & $\begin{array}{c}10.48 \\
E\end{array}$ & $\begin{array}{c}11.21 \\
A\end{array}$ & $\begin{array}{c}11.08 \\
B\end{array}$ & $\begin{array}{c}10.74 \\
C\end{array}$ & $\begin{array}{c}10.56 \\
D\end{array}$ & & \\
\hline
\end{tabular}

Values followed by the same letter(s) are not significantly different at $5 \%$ level.

Table (3): Effect of PBZ and KNO3 on total soluble sugars content (mg/g F.W.) in leaves of Sidik mango cv. (first season).

\begin{tabular}{|c|c|c|c|c|c|c|c|c|}
\hline \multirow[b]{2}{*}{ Treatments } & \multirow{2}{*}{ Conc. } & \multicolumn{5}{|c|}{ Date of sampling } & \multirow{2}{*}{ Mean } & \multirow{2}{*}{ Mean } \\
\hline & & Nov. & Dec. & Jan. & Feb. & Mar. & & \\
\hline \multirow{3}{*}{ PBZ (g/tree) } & 2.5 & $\begin{array}{c}11.25 \\
\mathrm{mn}\end{array}$ & $\begin{array}{c}11.86 \\
\mathrm{fg}\end{array}$ & $\begin{array}{c}11.36 \\
\mathrm{kl}\end{array}$ & $\begin{array}{c}11.00 \\
\text { rs }\end{array}$ & $\begin{array}{c}10.82 \\
u\end{array}$ & $\begin{array}{c}11.26 \\
D\end{array}$ & \multirow{3}{*}{$\begin{array}{c}11.62 \\
\mathbf{A}\end{array}$} \\
\hline & 5 & $\begin{array}{c}11.48 \\
\text { ii }\end{array}$ & 11.95 & $\begin{array}{c}11.81 \\
\mathrm{q}\end{array}$ & $\begin{array}{c}11.06 \\
p-r\end{array}$ & 11.00 & $\begin{array}{c}11.46 \\
\mathrm{C}\end{array}$ & \\
\hline & 7.5 & $\begin{array}{c}12.06 \\
C\end{array}$ & $\begin{array}{c}12.47 \\
a\end{array}$ & $\begin{array}{c}12.31 \\
b\end{array}$ & $\begin{array}{c}12.03 \\
\mathrm{~cd}\end{array}$ & $\begin{array}{c}11.84 \\
\mathrm{fg}\end{array}$ & $\begin{array}{c}12.14 \\
A\end{array}$ & \\
\hline \multirow{3}{*}{ KNO3 (\%) } & & $\begin{array}{c}11.05 \\
\text { p-r }\end{array}$ & $\begin{array}{c}11.38 \\
\mathrm{kl}\end{array}$ & $\begin{array}{c}11.21 \\
\mathrm{n}\end{array}$ & $\begin{array}{c}11.12 \\
\text { op }\end{array}$ & $\begin{array}{c}10.85 \\
\text { tu }\end{array}$ & $\begin{array}{c}11.12 \\
F\end{array}$ & \multirow{3}{*}{$\begin{array}{c}11.41 \\
\text { B }\end{array}$} \\
\hline & 2 & $\begin{array}{c}11.26 \\
\mathrm{mn}\end{array}$ & $\begin{array}{c}11.91 \\
\text { ef }\end{array}$ & $\begin{array}{c}11.62 \\
\mathrm{~h}\end{array}$ & $\begin{array}{c}11.41 \\
\mathrm{jk}\end{array}$ & $\begin{array}{c}11.20 \\
\text { no }\end{array}$ & $\begin{array}{c}11.48 \\
\text { C }\end{array}$ & \\
\hline & & $\begin{array}{c}11.32 \\
\text { Im }\end{array}$ & $\begin{array}{c}12.11 \\
\mathrm{C}\end{array}$ & $\begin{array}{c}11.85 \\
\mathrm{fg}\end{array}$ & $\begin{array}{c}11.62 \\
\mathrm{~h}\end{array}$ & $\begin{array}{l}11.31 \\
\mathrm{Im}\end{array}$ & $\begin{array}{c}11.64 \\
\text { B }\end{array}$ & \\
\hline \multicolumn{2}{|c|}{ Control } & $\begin{array}{c}11.03 \\
\mathrm{qr}\end{array}$ & $\begin{array}{c}11.51 \\
i\end{array}$ & $\begin{array}{c}11.26 \\
\mathrm{mn}\end{array}$ & $\begin{array}{c}11.09 \\
\mathrm{pq}\end{array}$ & $\begin{array}{c}10.93 \\
\text { st }\end{array}$ & $\begin{array}{c}11.16 \\
\mathrm{E}\end{array}$ & $\begin{array}{c}11.16 \\
\mathbf{C}\end{array}$ \\
\hline \multicolumn{2}{|l|}{ Mean } & $\begin{array}{c}11.36 \\
C\end{array}$ & $\begin{array}{c}11.88 \\
\boldsymbol{A}\end{array}$ & $\begin{array}{c}11.63 \\
B\end{array}$ & $\begin{array}{c}11.33 \\
C\end{array}$ & $\begin{array}{c}11.13 \\
D\end{array}$ & & \\
\hline
\end{tabular}

Values followed by the same letter(s) are not significantly different at $5 \%$ level. 
Table (4): Effect of PBZ and KNO3 on total soluble sugars content (mg/g F.W.) in leaves of Sidik mango cv. (second season).

\begin{tabular}{|c|c|c|c|c|c|c|c|c|}
\hline \multirow{2}{*}{ Treatments } & \multirow{2}{*}{ Conc. } & \multicolumn{5}{|c|}{ Date of sampling } & \multirow{2}{*}{ Mean } & \multirow{2}{*}{ Mean } \\
\hline & & Nov. & Dec. & Jan. & Feb. & Mar. & & \\
\hline \multirow{3}{*}{ PBZ (g/tree) } & 2.5 & $\begin{array}{c}12.15 \\
\mathrm{pq}\end{array}$ & $\begin{array}{c}12.93 \\
\mathrm{i}-\mathrm{I}\end{array}$ & $\begin{array}{c}12.81 \\
\text { Im }\end{array}$ & $\begin{array}{c}12.46 \\
\text { no }\end{array}$ & $\begin{array}{c}12.19 \\
p\end{array}$ & $\begin{array}{c}12.51 \\
D\end{array}$ & \multirow{3}{*}{$\begin{array}{c}12.96 \\
\mathbf{A}\end{array}$} \\
\hline & 5 & $\begin{array}{c}12.98 \\
h-j\end{array}$ & $\begin{array}{c}13.36 \\
d\end{array}$ & $\begin{array}{c}13.06 \\
\text { gh }\end{array}$ & $\begin{array}{c}12.85 \\
\mathrm{kl}\end{array}$ & $\begin{array}{c}12.43 \\
\text { no }\end{array}$ & $\begin{array}{c}12.93 \\
\mathrm{C}\end{array}$ & \\
\hline & 7.5 & $\begin{array}{c}13.52 \\
c\end{array}$ & $\begin{array}{c}13.93 \\
a\end{array}$ & $\begin{array}{c}13.65 \\
b\end{array}$ & $\begin{array}{c}13.22 \\
b\end{array}$ & $\begin{array}{c}13.00 \\
\text { de }\end{array}$ & $\begin{array}{c}13.46 \\
A\end{array}$ & \\
\hline \multirow{3}{*}{ KNO3 (\%) } & 1 & $\begin{array}{c}12.00 \\
r\end{array}$ & $\begin{array}{c}12.86 \\
j-1\end{array}$ & $\begin{array}{c}12.39 \\
\text { no }\end{array}$ & $\begin{array}{c}12.21 \\
p\end{array}$ & $\begin{array}{c}12.05 \\
\text { qr }\end{array}$ & $\begin{array}{c}12.30 \\
E\end{array}$ & \multirow{3}{*}{$\begin{array}{c}12.83 \\
\text { B }\end{array}$} \\
\hline & 2 & $\begin{array}{c}12.86 \\
\mathrm{j}-\mathrm{I}\end{array}$ & $\begin{array}{c}13.81 \\
a \\
\end{array}$ & $\begin{array}{c}13.02 \\
\mathrm{hi}\end{array}$ & $\begin{array}{c}12.82 \\
\mathrm{k}-\mathrm{m}\end{array}$ & $\begin{array}{c}12.18 \\
P\end{array}$ & $\begin{array}{c}12.94 \\
\mathrm{C}\end{array}$ & \\
\hline & 4 & $\begin{array}{c}13.21 \\
\text { ef }\end{array}$ & $\begin{array}{c}13.65 \\
b\end{array}$ & $\begin{array}{c}13.32 \\
\text { de }\end{array}$ & $\begin{array}{c}13.15 \\
\mathrm{fg}\end{array}$ & $\begin{array}{c}12.94 \\
\mathrm{~h}-\mathrm{k}\end{array}$ & $\begin{array}{c}13.25 \\
B\end{array}$ & \\
\hline \multicolumn{2}{|c|}{ Control } & $\begin{array}{c}12.36 \\
0\end{array}$ & $\begin{array}{c}12.92 \\
i-I\end{array}$ & $\begin{array}{c}12.71 \\
\mathrm{~m}\end{array}$ & $\begin{array}{c}12.49 \\
n\end{array}$ & $\begin{array}{c}12.16 \\
\mathrm{pq}\end{array}$ & $\begin{array}{c}12.53 \\
D\end{array}$ & $\begin{array}{c}12.53 \\
\text { C }\end{array}$ \\
\hline \multicolumn{2}{|l|}{ Mean } & $\begin{array}{c}12.72 \\
D\end{array}$ & $\begin{array}{c}13.35 \\
\boldsymbol{A}\end{array}$ & $\begin{array}{c}12.99 \\
B\end{array}$ & $\begin{array}{c}12.74 \\
C\end{array}$ & $\begin{array}{c}12.42 \\
E\end{array}$ & & \\
\hline
\end{tabular}

Values followed by the same letter(s) are not significantly different at $5 \%$ level.

\subsection{Effect of PBZ and KNO3 on total soluble amino acids contents in leaves of Ewais and Sidik mangos:}

Tables (5, 6,7 and 8) clear an obvious increase in amino acid content in the leaves of trees treated with PBZ or KNO3 than the control. Differences between the contents of treated trees and untreated ones were significant.

Total amino acids content in leaves of Ewais cultivar showed a pronounced and significant increase in this content with increasing PBZ concentration. Interaction between PBZ concentration and date revealed a significant increase in this content in Dec., then showed continuous decrease from Dec. up to Mar. This trend was observed with all treated concentrations and control trees.

In March, the highest amino acid content was recorded in the leaves of trees received the highest PBZ dose. This was obvious in both seasons. Concerning Sidik cultivar the same trend was noticed with PBZ treatments. The interaction between tested concentrations and dates of application also showed the same trend and changes that described above with Ewais.

Application of $\mathrm{KNO} 3$ significantly increased leaves amino acids content compared to the control, this was noticed in both cultivars. A direct relationship was found between the used concentrations and this content.

Concerning the changes in amino acids content in the leaves during the sampling dates it showed significant increase from Nov. to Dec. as it attained the maximum level during Dec. A significant fall in this content was detected during the period from Jan. to Mar.(bud break). This trend was confirmed in both seasons for the two tested cultivars. Concerning cultivars differences in this concern, leaves of Sidik recorded higher amino acids content than Ewais which may correlated with the early flower bud initiation and bud break for Sedik than Ewais. Supporting to the above mentioned results Patil et al (1988) reported that before and during flower bud differentiation (FBD) stages 
there was a significant decrease in the total free amino acids content of the bud and it was considerably lower than that of scar buds. The total free amino acids content of differentiated buds was significantly higher than that of scar and undifferentiated buds. Arginine and Phenylalanine increased enormously during FBD. Moreover, Abass and Mehana (1995) suggested that flower bud formation in mango trees possibly associated with the metabolites of amino acids in shoots of Hindi Khassa and Zebda mango cultivars.

Table (5): Effect of PBZ and $\mathrm{KNO} 3$ on total soluble amino acids content ( $\mathrm{mg} / 100 \mathrm{~g}$ F.W.) in leaves of Ewais mango cv. (first season).

\begin{tabular}{|c|c|c|c|c|c|c|c|c|}
\hline \multirow{2}{*}{ Treatments } & \multirow{2}{*}{$\begin{array}{c}\text { Con } \\
\text { c. }\end{array}$} & \multicolumn{5}{|c|}{ Date of sampling } & \multirow{2}{*}{ Mean } & \multirow{2}{*}{ Mean } \\
\hline & & Nov. & Dec. & Jan. & Feb. & Mar. & & \\
\hline \multirow{3}{*}{$\begin{array}{c}\text { PBZ } \\
\text { (g/tree) }\end{array}$} & 2.5 & $\underset{t}{25.18}$ & $\begin{array}{c}35.22 \\
C\end{array}$ & $\underset{i}{30.15}$ & $\begin{array}{c}27.16 \\
p\end{array}$ & $\begin{array}{c}25.36 \\
s\end{array}$ & $\begin{array}{c}28.61 \\
\text { D }\end{array}$ & \multirow{3}{*}{$\begin{array}{c}30.90 \\
\mathbf{A}\end{array}$} \\
\hline & 5 & $\begin{array}{c}29.31 \\
1\end{array}$ & $\begin{array}{c}36.11 \\
b\end{array}$ & $\begin{array}{c}32.61 \\
\mathrm{e}\end{array}$ & $\begin{array}{c}30.11 \\
\mathrm{i}\end{array}$ & $\begin{array}{c}28.12 \\
0\end{array}$ & $\begin{array}{c}31.25 \\
B\end{array}$ & \\
\hline & 7.5 & $\begin{array}{c}31.23 \\
h\end{array}$ & $\begin{array}{c}39.41 \\
a\end{array}$ & $\begin{array}{c}33.24 \\
d\end{array}$ & $\begin{array}{c}31.35 \\
\mathrm{~g}\end{array}$ & $\begin{array}{c}29.05 \\
m\end{array}$ & $\begin{array}{c}32.85 \\
\text { A }\end{array}$ & \\
\hline \multirow{3}{*}{ KNO3 (\%) } & 1 & $\begin{array}{c}23.41 \\
u\end{array}$ & $\begin{array}{c}31.26 \\
\text { gh }\end{array}$ & $\begin{array}{c}29.11 \\
\mathrm{~m}\end{array}$ & $\begin{array}{c}27.16 \\
p\end{array}$ & $\begin{array}{c}23.18 \\
v\end{array}$ & $\begin{array}{c}26.82 \\
F\end{array}$ & \multirow{3}{*}{$\begin{array}{c}28.22 \\
\text { B }\end{array}$} \\
\hline & 2 & $\begin{array}{c}26.82 \\
q\end{array}$ & $\begin{array}{c}32.00 \\
f\end{array}$ & $\begin{array}{c}30.00 \\
j\end{array}$ & $\begin{array}{c}26.18 \\
r\end{array}$ & $\begin{array}{c}25.14 \\
t\end{array}$ & $\begin{array}{c}28.03 \\
E\end{array}$ & \\
\hline & 4 & $\begin{array}{c}28.31 \\
n\end{array}$ & $\begin{array}{c}33.17 \\
d\end{array}$ & $\begin{array}{c}31.18 \\
h\end{array}$ & $\begin{array}{c}29.26 \\
1\end{array}$ & $\begin{array}{c}27.12 \\
p\end{array}$ & $\begin{array}{c}29.81 \\
\text { C }\end{array}$ & \\
\hline \multicolumn{2}{|c|}{ Control } & $\begin{array}{c}22.36 \\
w\end{array}$ & $\begin{array}{c}28.16 \\
0\end{array}$ & $\begin{array}{c}29.86 \\
k\end{array}$ & $\begin{array}{c}26.25 \\
r\end{array}$ & $\begin{array}{c}23.12 \\
v\end{array}$ & $\begin{array}{c}25.95 \\
G\end{array}$ & $\begin{array}{c}25.95 \\
\text { C }\end{array}$ \\
\hline \multicolumn{2}{|c|}{ Mean } & $\begin{array}{c}26.66 \\
D\end{array}$ & $\begin{array}{c}33.61 \\
\boldsymbol{A}\end{array}$ & $\begin{array}{c}30.87 \\
\text { B }\end{array}$ & $\begin{array}{c}28.21 \\
C\end{array}$ & $\begin{array}{c}25.87 \\
E\end{array}$ & & \\
\hline
\end{tabular}

Values followed by the same letter(s) are not significantly different at $5 \%$ level.

Table (6): Effect of PBZ and $\mathrm{KNO}$ on total soluble amino acids content

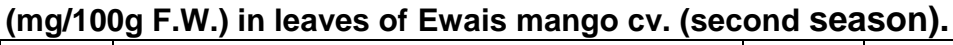

\begin{tabular}{|c|c|c|c|c|c|c|c|c|}
\hline \multirow{2}{*}{ Treatments } & \multirow{2}{*}{ Conc. } & \multicolumn{5}{|c|}{ Date of sampling } & \multirow{2}{*}{ Mean } & \multirow{2}{*}{ Mean } \\
\hline & & Nov. & Dec. & Jan. & Feb. & Mar. & & \\
\hline \multirow{3}{*}{ PBZ (g/tree) } & 2.5 & $\begin{array}{c}26.08 \\
\text { rs }\end{array}$ & $\begin{array}{c}28.41 \\
\text { | }\end{array}$ & 28.06 & $\begin{array}{c}27.55 \\
k\end{array}$ & $\begin{array}{c}26.32 \\
p\end{array}$ & $\begin{array}{c}27.28 \\
E\end{array}$ & \multirow{3}{*}{$\begin{array}{c}29.64 \\
\text { A }\end{array}$} \\
\hline & 5 & $\begin{array}{c}27.18 \\
m\end{array}$ & $\begin{array}{c}30.18 \\
\mathrm{e}\end{array}$ & $\begin{array}{c}29.91 \\
f\end{array}$ & $\begin{array}{c}29.32 \\
g\end{array}$ & $\begin{array}{c}28.89 \\
h\end{array}$ & $\begin{array}{c}29.09 \\
\text { B }\end{array}$ & \\
\hline & 7.5 & $\underset{j}{28.12}$ & $\begin{array}{c}35.38 \\
a\end{array}$ & $\begin{array}{c}34.82 \\
b\end{array}$ & $\begin{array}{c}32.86 \\
c\end{array}$ & $\begin{array}{c}31.65 \\
d\end{array}$ & $\begin{array}{c}32.56 \\
\text { A }\end{array}$ & \\
\hline \multirow{3}{*}{ KNO3 (\%) } & 1 & $\begin{array}{c}25.05 \\
t\end{array}$ & $\begin{array}{c}27.41 \\
\text { i }\end{array}$ & $\begin{array}{c}27.36 \\
\mid\end{array}$ & $\begin{array}{c}26.93 \\
n\end{array}$ & $\begin{array}{c}26.72 \\
0\end{array}$ & $\begin{array}{c}26.69 \\
F\end{array}$ & \multirow{3}{*}{$\begin{array}{c}27.34 \\
\text { B }\end{array}$} \\
\hline & 2 & $\begin{array}{c}26.00 \\
\mathrm{~s}\end{array}$ & $\begin{array}{c}28.14 \\
\mathrm{~J}\end{array}$ & $\underset{j}{28.12}$ & $\begin{array}{c}27.62 \\
k\end{array}$ & $\begin{array}{c}27.00 \\
n\end{array}$ & $\begin{array}{c}27.37 \\
\text { D }\end{array}$ & \\
\hline & 4 & $\begin{array}{c}26.14 \\
\mathrm{gr}\end{array}$ & $\begin{array}{c}29.36 \\
\mathrm{~g}\end{array}$ & $\begin{array}{c}29.00 \\
h\end{array}$ & $\underset{j}{28.06}$ & $\begin{array}{c}27.36 \\
\mid\end{array}$ & $\begin{array}{c}27.98 \\
\text { C }\end{array}$ & \\
\hline \multicolumn{2}{|c|}{ Control } & $\begin{array}{c}24.81 \\
u\end{array}$ & $\begin{array}{c}26.32 \\
p\end{array}$ & $\begin{array}{c}26.21 \\
\mathrm{pq}\end{array}$ & $\begin{array}{c}26.11 \\
q-s\end{array}$ & $\begin{array}{c}25.00 \\
t\end{array}$ & $\begin{array}{c}25.69 \\
G\end{array}$ & $\begin{array}{c}25.69 \\
\text { C }\end{array}$ \\
\hline \multicolumn{2}{|l|}{ Mean } & $\begin{array}{c}26.19 \\
\text { E }\end{array}$ & $\begin{array}{c}29.31 \\
\text { A }\end{array}$ & $\begin{array}{c}29.06 \\
\text { B }\end{array}$ & $\begin{array}{c}28.35 \\
\text { C }\end{array}$ & $\begin{array}{c}27.56 \\
\text { D }\end{array}$ & & \\
\hline
\end{tabular}

Values followed by the same letter(s) are not significantly different at $5 \%$ level. 
J. Agric. Sci. Mansoura Univ., 32 (6), June, 2007

Table (7): Effect of PBZ and KNO3 on total soluble amino acids content

\begin{tabular}{|c|c|c|c|c|c|c|c|c|}
\hline \multirow{2}{*}{ Treatments } & \multirow{2}{*}{ Conc. } & \multicolumn{5}{|c|}{ Date of sampling } & \multirow[b]{2}{*}{ Mean } & \multirow[b]{2}{*}{ Mean } \\
\hline & & Nov. & Dec. & Jan. & Feb. & Mar. & & \\
\hline \multirow{3}{*}{ PBZ (g/tree) } & 2.5 & $\begin{array}{c}47.00 \\
\text { ) }\end{array}$ & $\begin{array}{c}52.12 \\
\mathrm{C}\end{array}$ & $\begin{array}{c}46.18 \\
m\end{array}$ & $\begin{array}{c}43.12 \\
\mathrm{q}\end{array}$ & $\begin{array}{c}42.39 \\
\text { rs }\end{array}$ & $\begin{array}{c}46.16 \\
\text { C }\end{array}$ & \multirow{3}{*}{$\begin{array}{c}48.33 \\
\text { A }\end{array}$} \\
\hline & 5 & $\begin{array}{c}47.21 \\
k\end{array}$ & $\begin{array}{c}53.46 \\
b\end{array}$ & $\begin{array}{c}48.31 \\
\mathrm{~g}\end{array}$ & $\begin{array}{c}48.05 \\
\mathrm{ij}\end{array}$ & $\begin{array}{c}47.32 \\
k\end{array}$ & $\begin{array}{c}48.87 \\
\text { B }\end{array}$ & \\
\hline & 7.5 & $\begin{array}{c}48.36 \\
\mathrm{~g}\end{array}$ & $\begin{array}{c}55.91 \\
\text { a }\end{array}$ & $\begin{array}{c}49.06 \\
\mathrm{e}\end{array}$ & $\underset{f}{48.61}$ & 47.92 & $\begin{array}{c}49.97 \\
\text { A }\end{array}$ & \\
\hline \multirow{3}{*}{ KNO3 (\%) } & 1 & $\begin{array}{c}42.05 \\
t\end{array}$ & $\begin{array}{c}48.16 \\
\mathrm{hi}\end{array}$ & $\begin{array}{c}46.18 \\
m\end{array}$ & $\begin{array}{c}43.18 \\
q\end{array}$ & $\begin{array}{c}42.36 \\
\mathrm{~s}\end{array}$ & $\begin{array}{c}44.38 \\
F\end{array}$ & \multirow{3}{*}{$\begin{array}{c}45.13 \\
\text { B }\end{array}$} \\
\hline & 2 & $\begin{array}{c}42.19 \\
r\end{array}$ & $\begin{array}{c}49.00 \\
\mathrm{e}\end{array}$ & $\begin{array}{c}47.32 \\
k\end{array}$ & $\begin{array}{c}44.00 \\
0\end{array}$ & $\begin{array}{c}43.62 \\
p\end{array}$ & $\begin{array}{c}45.22 \\
E\end{array}$ & \\
\hline & 4 & $\begin{array}{c}43.62 \\
p\end{array}$ & $\begin{array}{c}49.36 \\
d\end{array}$ & $\begin{array}{c}48.25 \\
\text { gh }\end{array}$ & $\begin{array}{c}45.64 \\
n\end{array}$ & $\begin{array}{c}42.11 \\
t\end{array}$ & $\begin{array}{c}45.79 \\
D\end{array}$ & \\
\hline \multicolumn{2}{|c|}{ Control } & $\begin{array}{c}39.25 \\
v\end{array}$ & $\begin{array}{c}42.18 \\
t\end{array}$ & $\begin{array}{c}41.33 \\
u\end{array}$ & $\begin{array}{c}39.16 \\
V\end{array}$ & $\begin{array}{c}32.41 \\
\text { w }\end{array}$ & $\begin{array}{c}38.86 \\
G\end{array}$ & $\begin{array}{c}38.86 \\
\text { C }\end{array}$ \\
\hline \multicolumn{2}{|c|}{ Mean } & $\begin{array}{c}44.24 \\
\text { D }\end{array}$ & $\begin{array}{c}50.02 \\
\boldsymbol{A}\end{array}$ & $\begin{array}{c}46.66 \\
\text { B }\end{array}$ & $\begin{array}{c}44.53 \\
C\end{array}$ & $\begin{array}{c}42.59 \\
E\end{array}$ & & \\
\hline
\end{tabular}

Values followed by the same letter(s) are not significantly different at $5 \%$ level.

Table (8): Effect of PBZ and KNO3 on total soluble amino acids content

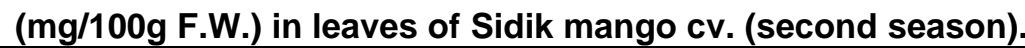

\begin{tabular}{|c|c|c|c|c|c|c|c|c|}
\hline \multirow{2}{*}{ Treatments } & \multirow{2}{*}{ Conc. } & \multicolumn{5}{|c|}{ Date of sampling } & \multirow{2}{*}{ Mean } & \multirow{2}{*}{ Mean } \\
\hline & & Nov. & Dec. & Jan. & Feb. & Mar. & & \\
\hline \multirow{3}{*}{ PBZ (g/tree) } & 2.5 & $\begin{array}{c}49.63 \\
r\end{array}$ & $\begin{array}{c}55.16 \\
\mathrm{e}\end{array}$ & $\begin{array}{c}51.05 \\
\mathrm{~m}\end{array}$ & $\begin{array}{c}48.11 \\
t\end{array}$ & $\begin{array}{c}41.18 \\
\backslash\end{array}$ & $\begin{array}{c}49.02 \\
F\end{array}$ & \multirow{3}{*}{$\begin{array}{c}51.29 \\
\text { B }\end{array}$} \\
\hline & 5 & $\begin{array}{c}50.22 \\
0\end{array}$ & $\begin{array}{c}56.42 \\
b\end{array}$ & $\begin{array}{c}53.16 \\
h\end{array}$ & $\begin{array}{c}49.92 \\
q\end{array}$ & $\begin{array}{c}46.71 \\
\mathrm{~V}\end{array}$ & $\begin{array}{c}51.28 \\
D\end{array}$ & \\
\hline & 7.5 & $\begin{array}{c}55.33 \\
d\end{array}$ & $\begin{array}{c}58.19 \\
a\end{array}$ & $\begin{array}{c}54.22 \\
\mathrm{~g}\end{array}$ & $\begin{array}{c}50.12 \\
\text { op }\end{array}$ & $\begin{array}{c}50.05 \\
p\end{array}$ & $\begin{array}{c}53.58 \\
\text { A }\end{array}$ & \\
\hline \multirow{3}{*}{ KNO3 (\%) } & 1 & $\begin{array}{c}51.22 \\
1\end{array}$ & $\begin{array}{c}54.12 \\
\mathrm{~g}\end{array}$ & $\begin{array}{c}52.14 \\
j\end{array}$ & $\begin{array}{c}46.19 \\
w\end{array}$ & $\begin{array}{c}44.32 \\
x\end{array}$ & $\begin{array}{c}49.60 \\
E\end{array}$ & \multirow{3}{*}{$\begin{array}{c}51.71 \\
\text { A }\end{array}$} \\
\hline & 2 & $\begin{array}{c}52.33 \\
i\end{array}$ & $\begin{array}{c}55.22 \\
\text { de }\end{array}$ & $\begin{array}{c}53.05 \\
h\end{array}$ & $\begin{array}{c}50.91 \\
n\end{array}$ & $\begin{array}{c}48.62 \\
s\end{array}$ & $\begin{array}{c}52.02 \\
\text { C }\end{array}$ & \\
\hline & 4 & $\begin{array}{c}54.66 \\
f\end{array}$ & $\begin{array}{c}55.89 \\
C\end{array}$ & $\begin{array}{c}55.11 \\
\mathrm{e}\end{array}$ & $\begin{array}{c}51.82 \\
k\end{array}$ & $\begin{array}{c}50.08 \\
p\end{array}$ & $\begin{array}{c}53.51 \\
\text { B }\end{array}$ & \\
\hline \multicolumn{2}{|c|}{ Control } & $\begin{array}{c}43.76 \\
y\end{array}$ & $\begin{array}{c}46.82 \\
u\end{array}$ & $\begin{array}{c}44.32 \\
x \\
\end{array}$ & $\begin{array}{c}42.36 \\
z\end{array}$ & 41.75 & $\begin{array}{c}43.80 \\
G\end{array}$ & $\begin{array}{c}43.80 \\
\text { C }\end{array}$ \\
\hline \multicolumn{2}{|l|}{ Mean } & $\begin{array}{c}51.02 \\
C\end{array}$ & $\begin{array}{c}54.54 \\
A\end{array}$ & $\begin{array}{c}51.86 \\
B\end{array}$ & $\begin{array}{c}47.62 \\
D\end{array}$ & $\begin{array}{c}46.10 \\
E\end{array}$ & & \\
\hline
\end{tabular}

Values followed by the same letter(s) are not significantly different at $5 \%$ level. 


\section{REFERENCES}

Anez,M. (2004). Effect of thiosulphates and paclobutrazol on type and length of branch in mango Haden. Acta Hort. 645: 209-215.

A.O.A.C. (1975): Official Methods of Analysis of the Association of Official Agricultural Chemists. Washington D.C. $12^{\text {th }}$ ed.

Abbas, M.T. and S.A. Mehana (1995): Biochemical changes of some endogenous components in mango shoots to alternate bearing. Zagazig J. Agric. Res., 22: 869-880.

Duncan, B.D.(1955). Multiple range and multiple $F$ tests. Biometrics, 11:1-42.

Khattab M .M., Haseeb G.M.M. , Shaban A.E. and M.A. Arafa (2006). Effect of Paclobutrazol and Potassium nitrate on flowering and fruiting of Ewais and Sidik mango trees. Bull. Fac.Agric. Cairo Univ., 57 (1):107 124.

Oosthuyse, S.A. (1993). Effect of spray application of KNO3, urea and growth regulators on the yield of Tommy Atkins mango. Yearbook- South African Mango Growers. Association, 13:5 8-62.

Oosthuyse, S.A. (1996). Effect of KNO3 spray to flowering mango trees on fruit retention, fruit size, tree yield and fruit quality. Yearbook- South African Mango Growers. Association, 16:27-31

Patil, P.B.; M.M. Rao; P.W. Basarkar; K.V. Janardhan; C.N. Srinivasan and U.G. Nalawadi, (1988). Role of free amino acids in fruit bud differentiation in Alphonso mango shoots. Acta Hort.231:405-411.

Rosein, H.(1957). A modified ninhydrine colorimetric analysis for amino acids. Arch. Biochem. Biophys. 67: $10-15$.

Shaban, A.E.A (2004). Effect of Ethrel spraying on inducing flowering in the off year of mango trees. Annals Agric., Ain shams Univ., Cairo, 49(2), 687-698.

Sharma, T.R.; P.K. Nair and M.K. Nema (1990). Effect of foliar spray of urea, $\mathrm{KNO} 3$ and NAA on fruiting behavior of mango cv. Langra. Orrisa Journal of Hort.18(1): 42-47.

Snedecor, G.W. and W.G. Cochran (1980). Statistical Methods. 7 th Ed. lowa State Univ. Press, Ames, lowa, U.S.A.,p.507.

Tongumpai, P. N.; Hongsbhanich and V. C. Hoi (1989). Cultar for flowering regulation of mango in Thailand. Acta Hort., 239:375-378.

Tongumpai, P.; Chantakulchan, K.; Subhadrabandhu, S.; Ogata, R.(1997):Foliar application of paclobutrazol on flowering of mango. Acta Horticulturae. 455, 175-179.

Zidan, Z.; I. Shawky and D. Dahshan (1975). Flower bud induction in mango trees. Annals Agric. Sci. Ain Shams Univ., Cairo, 20: 151-158. 


\section{تأثثر الباكلوبترازول ونترات البوتاسيوم على الحالة الغذائية لأوراق أشجار المـانجو عويس وصديق الباكيز \\ 1- 1 السكريات والأحماض الأمينية الكلية

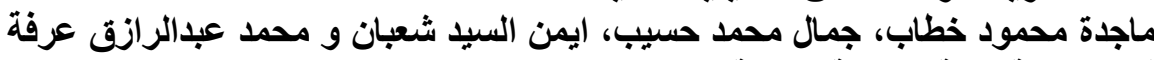

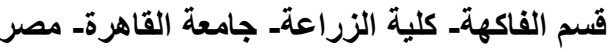

أجريت هذه الدراسة خلال موسمين على أنثجار مانجو مطعومة صنفى صديق و عويس نامية فى منطقة

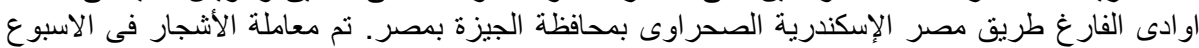
الأول من اكتوبر طريق

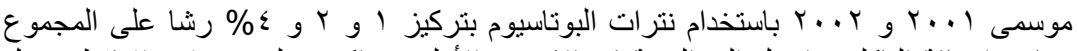

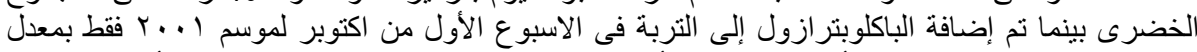

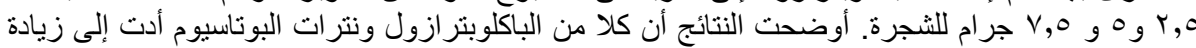

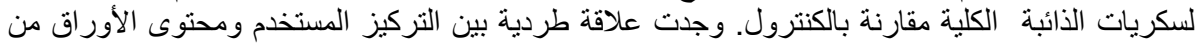

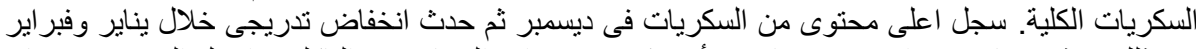

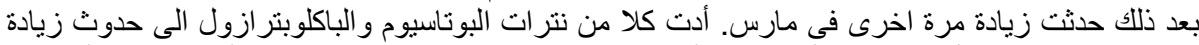

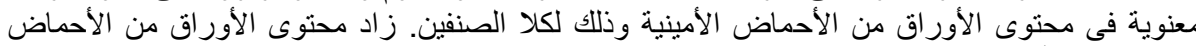

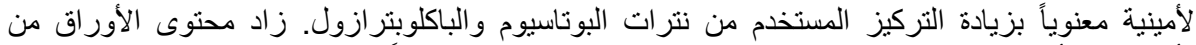

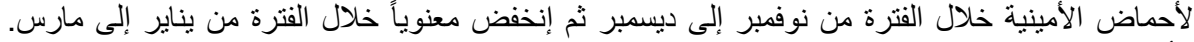

وتأكد ذلك مع كلا الصنفين خلال موسمى الدراسة. 